\title{
A streakline method for computing two-dimensional vortical flows
}

\author{
Surya Prasad G. Dinavahi \\ Department of Naval Architecture and Marine Engineering, The University of Michigan, Ann Arbor, MI, USA
}

\begin{abstract}
Dinavahi, S.P.G., A strcakline method for computing two-dimensional vortical flows, Applicd Numerical Mathematics 11 (1993) 283-308.

A forward time-stepping method is developed for computing free-streamline flows past two-dimensional bodies. The field vorticity is represented by vortex sheets. The kinematic boundary condition of no flow across the field vortex sheets is satisfied temporally by shifting the sheets normal to themselves as dictated by the local field velocities. The Helmholtz vorticity equation is reformulated as a dynamic boundary condition to be applied along the field vortex sheets. It is demonstrated that for a specified separation point, the solution evolves toward the Kirchhoff steady free-streamline flow. It is further demonstrated that in shedding vortex sheets from both corners of a polygonal contour with two corners, the method picks the forward corner as the separation point. For a circular polygonal contour with eleven corner points, five of which are allowed to shed vortex sheets, the method picks the corner point at 54 degrees from the forward stagnation point as the separation point. It is observed that this is close to 55.04 degrees, which is the forwardmost separation point allowed by the classical free-streamline theory for the circle.
\end{abstract}

\section{Introduction}

A new method is developed for arriving at stationary inviscid vortical flows, starting from the attached potential flow as the initial condition. A vortical flow as referred to in this work is one where a body is fixed in an otherwise uniform stream of such high Reynolds number that the vorticity that is present in the field and on the body is in the form of infinitesimally thin sheets. Here, the boundary layer is detached from the body and its vorticity is convected at order-one distances from the body along free vortex sheets, unlike the flow within an attached boundary layer. This is a case where convection overwhelms diffusion. The present study is confined to two dimensions.

The model consists of a bound vortex sheet on the body contour and free vortex sheets in the field, which are connected to the body contour at the separation points. The end results of computation by this new method are, conceptually, the classical Kirchhoff open free-streamline 
flows, where the free streamlines are also vortex sheets. In essence, the method to be outlined and demonstrated computes the steady Kirchhoff free-streamline flows as the steady-state limit of an initial value problem.

Analytic solutions for stationary free-streamline flows past flat plates and polygonal sections have existed for some time. The formulation is Eulerian and the solution procedure involves conformal mapping techniques in which the physical plane and the hodograph plane are mapped conformally onto a parametric plane where the mixed boundary value problem is solved. In the case of bodies with sharp corners the separation points, which are at the corners, are known a priori. The solution procedure for the free-streamline flow past curved bodies of arbitrary section shape was first proposed by Levi-Civita [6] in 1907. The case of circular and elliptic cylinders placed symmetrical in a uniform stream was studied by Brodetsky [2] in 1923, Ford [4] in 1928, and Schmieden [11] in 1929. Birkhoff [1] gives a very comprehensive review of the solution procedure for flow past curved sections of arbitrary shape and presents some of the results obtained by solving the resulting nonlinear equations numerically.

In the present work, the free-streamline problem is formulated as an initial value problem. The initial value procedure developed in this work is applied in investigating the possibility of predicting definite ideal flow separation points on general polygonal contours. First, the computation procedure is applied to simple shapes, and it is confirmed that the correct separation points are achieved as the computation proceeds in time. The procedure is then applied to a semi-circular polygonal contour with eleven corner points. The results are interesting.

The method developed specifically for the investigations described above can be classified as a vortex-fitting method. It is termed the "streakline method". This is because, with a streakline defined as the line connecting all the particles that have passed through a given point since the origin of time, this method computes the approximate evolution of streaklines with time. The streaklines are also the intersections of the two-dimensional solution plane with vortex sheets which carry vorticity that is shed at the body separation points. The generated streaklines are conveniently viewed as "vortex tubes" through which the vorticity flows. These are flexible but inextensible tubes that are convected normal to themselves by the local velocity field. However, these tubes (vortex sheets) are not free to drift in the field as they are connected to the body at the separation points. At each time step new segments are added at the downstream ends of the tubes as needed to prevent the vorticity from flowing out into the field. The flow of vorticity in the tubes is governed by the Helmholtz vorticity equation, which forms the dynamic boundary condition.

It is required in pursuing the objectives of this work that the time-stepping calculations be carried on to a relatively large time. In order to achieve this, it is necessary to avoid the vortex sheet roll-up and other small scale instabilities associated with the time-varying flow field. The difficulties in forward time-stepping the Euler equations toward steady-state solutions are well known. Procedures have been devised for suppressing the unwanted behavior; a recent method uses the concept of artificial compressibility in the incompressible flow problem formulation (Rizzi and Eriksson [9]). In the current work, a different approach is devised in developing a computational method that proceeds toward the steady average state free of the usual difficulties imposed by small scale instabilities and vortex sheet roll-up.

The general flow can be viewed as evolving on two scales in both length and time. The large scales represent the evolution of the gross features of the flow toward a steady average 
configuration. The aim of the present work is to retain this character of the flow field. The small scales, on the other hand, represent the local details in space and time that are so troublesome in efforts to compute the large scale character of interest. Here, the unwanted small scale effects are filtered out in the progression of the computation. The small time scale flow features are eliminated by an averaging filter which averages the velocity field, at each time step, over some number of preceding successive time steps. The number of averaging steps, which is equivalent to the filter bandwidth, is adjusted to suit the particular computation. The spatial smoothing is accomplished by computing the field velocities on a fixed spatial grid, and then interpolating back to the streaklines in imposing the boundary conditions in the convection stage of the calculation procedure. In general, the streakline method averages out the unwanted small scale behavior as it develops, rather than from the final statistically stationary flow field, as is typically performed with experimental data.

The streakline method was developed in [3]. A brief description of the procedure and results is presented in the following sections.

\section{Equations of motion}

\subsection{Problem definition}

Consider the general two-dimensional flow past a cylindrical body. The stream has uniform velocity $\vec{V}_{\infty}$ at infinity. The fluid domain is the region denoted as $A$, bounded internally by the body surface contour $C_{1}$ and externally by the circle $C_{\infty}$ of infinite radius. The governing equations are:

- Helmholtz's vorticity transport equation:

$$
\frac{\mathrm{D}}{\mathrm{D} T} \omega(x, y, t)=\frac{1}{\operatorname{Re}} \nabla^{2} \omega(x, y, t) ;
$$

- continuity of mass:

$$
\nabla \cdot \vec{V}=0
$$

- definition of vorticity:

$$
\nabla \times \vec{V}=\omega \hat{k},
$$

which must be satisfied in $A$, with boundary conditions:

$$
\begin{array}{ll}
\vec{V}=\overrightarrow{0} & \text { on } C_{\mathrm{b}}, \\
\vec{V}=\vec{V}_{\infty} & \text { on } C_{\infty} .
\end{array}
$$

In the above equations, $\omega(x, y, t)$ is the field vorticity, $\vec{V}(x, y, t)$ is the field velocity, $\nabla$ is the gradient operator, and Re is the Reynolds number. Assume that the stream $\vec{V}_{\infty}$ is started impulsively. At this instant, no vorticity has been diffused from the body surface. Hence the 
flow at this initial instant is entirely irrotational [7]. The attached potential flow is therefore the proper initial condition, i.e.,

$$
\vec{V}=\vec{V}_{\mathrm{P}} \text { at } t=0 .
$$

$\vec{V}_{\mathrm{P}}$ is the field velocity initially.

Integration of (3), subject to (2) and (5), gives the following expression for the field velocity:

$$
\vec{V}=\vec{V}_{\infty}+\iint_{A} \omega(\xi, \eta) \hat{k} \times \nabla_{p} G(p, q) \mathrm{d} A
$$

where

$$
\begin{aligned}
& G(p, q)=\frac{1}{2 \pi} \ln \sqrt{(x-\xi)^{2}+(y-\eta)^{2}} \\
& \nabla_{p}=\frac{\partial}{\partial x} \hat{i}+\frac{\partial}{\partial y} \hat{j} .
\end{aligned}
$$

$p(x, y)$ denotes a field point and $q(\xi, \eta)$ is a vorticity point.

Equations (1) and (7) with the boundary condition (4) and the initial condition (6) is the general formulation to be solved. Now assume that the Reynolds number is high enough such that, in any fixed field of vision, the body boundary layer appears as an infinitesimally thin layer coincident with the body contour. Hence the body surface is represented as a vortex sheet. The assumption of high Reynolds number allows the reduction of the general formulation to a generalized potential flow problem in which field vorticity is concentrated into thin layers. The flow is potential outside these thin layers, but there is a mechanism for producing vorticity at the solid boundaries. Assuming that the field vorticity is concentrated into infinitesimally thin sheets, it is represented as

$$
\omega(x, y, t)=\gamma_{q}^{\mathrm{f}}(s, t) \delta(n),
$$

where $\delta(n)$ is the Dirac delta function, $\gamma_{q}^{\mathrm{f}}$ in the vortex sheet strength per unit length, $s$ is the coordinate tangent to the vortex sheet, and $n$ is the normal coordinate. The curvilinear coordinates $s$ and $n$ are fixed in space at the given instant of time. There are $M$ such vortex sheets present in the field at a given time. The expression for $\omega$ from (10) is substituted into equation (7) and integrated across each of the $M$ vortex sheets along $n$. The total field velocity is the summation due to the $M$ vortex sheets present in the field and that due to the bound vortex sheet on the body. This is expressed from (7) and (10) as

$$
\vec{V}=\vec{V}_{\infty}+\oint_{C_{\mathrm{b}}} \gamma_{q}^{\mathrm{b}} \hat{k} \times \nabla_{p} G(p, q) \mathrm{d} s_{q}+\sum_{m=1}^{M} \int_{C_{m}} \gamma_{q}^{\mathrm{f}} \hat{k} \times \nabla_{p} G(p, q) \mathrm{d} s_{q} .
$$

The superscripts $\mathrm{b}$ and $\mathrm{f}$ for $\gamma_{q}$ refer to the body and the field vortex strengths, respectively. $C_{m}$ is the contour of the $m$ th vortex trailer.

\subsection{Boundary conditions on free vortex sheets}

In this paper, the terms "vortex sheet", "vortex trailer", "vortex tube", and "streakline" are all used synonymously. They all refer to the intersection of a three-dimensional vortex sheet 
with the two-dimensional solution domain. Actual "vortex lines" are perpendicular to the solution plane and extend to infinity in both directions.

The vorticity transport equation (1), which governs the vorticity development in the field, can now be reformulated as an equation that governs the distribution of vorticity along the free vortex sheets in the field. Equation (1) is transformed into the $s-n$ coordinate system, where $s$ and $n$ are the instantaneous tangent and normal directions to the field vortex sheet respectively, to obtain,

$$
\begin{aligned}
& \frac{\partial \omega}{\partial t}+\frac{v_{s}}{h_{1}} \frac{\partial \omega}{\partial s}+\frac{v_{n}}{h_{2}} \frac{\partial \omega}{\partial n} \\
& =\frac{1}{\operatorname{Re}} \frac{1}{h_{1} h_{2}}\left\{\frac{h_{2}}{h_{1}} \frac{\partial^{2} \omega}{\partial s^{2}}+\frac{\partial}{\partial s}\left(\frac{h_{2}}{h_{1}}\right) \frac{\partial \omega}{\partial s}+\frac{h_{1}}{h_{2}} \frac{\partial^{2} \omega}{\partial n^{2}}+\frac{\partial}{\partial n}\left(\frac{h_{1}}{h_{2}}\right) \frac{\partial \omega}{\partial n}\right\} .
\end{aligned}
$$

Here $\omega=\omega(s, n, t), h_{1}=1+n / \rho, h_{2}=1, v_{s}$ is the velocity along tangential direction $s, v_{n}$ is the velocity in the normal direction $n$, and $\rho$ is the local radius of curvature of the vortex sheet.

Similarly, the continuity equation (2) can be written in the curvilinear coordinates as

$$
\frac{1}{h_{1} h_{2}} \frac{\partial\left(h_{2} v_{s}\right)}{\partial s}+\frac{1}{h_{1} h_{2}} \frac{\partial\left(h_{1} v_{n}\right)}{\partial n}=0 .
$$

By (10) the field vorticity is concentrated into vortex sheets which are viewed as stationary instantaneously. Allowing the sheets to move with the fluid in the $n$ direction, through the time increment $\Delta t=t-t_{0}$, equation (10) takes the form

$$
\omega(s, n, t)=\gamma_{q}^{\mathrm{f}}(s, t) \delta(n-0) .
$$

By substituting the above form for $\omega$ in equation (12) and integrating across the vortex sheet, we obtain the vorticity equation along the vortex sheet:

$$
\frac{\partial \gamma_{q}^{\mathrm{f}}}{\partial t}+\frac{\partial\left(v_{s} \gamma_{q}^{\mathrm{f}}\right)}{\partial s}+\gamma_{q}^{\mathrm{f}} \frac{v_{n}}{\rho}=\frac{1}{\operatorname{Re}}\left(\frac{\partial^{2} \gamma_{q}^{\mathrm{f}}}{\partial s^{2}}+\frac{\gamma_{q}^{\mathrm{f}}}{\rho^{2}}\right) .
$$

As the Reynolds number $\mathrm{Re} \rightarrow \infty$, the above equation reduces to

$$
\frac{\partial \gamma_{q}^{\mathrm{f}}}{\partial t}+\frac{\partial\left(v_{s} \gamma_{q}^{\mathrm{f}}\right)}{\partial s}+\gamma_{q}^{\mathrm{f}} \frac{v_{n}}{\rho}=0
$$

This is the vorticity transport equation governing the flow of vorticity along the vortex sheet and can be viewed as a dynamic boundary condition to be satisfied on each of the $M$ vortex sheets implied in (11).

The $M$ vortex sheets that are present in the field are material surfaces. Further, each vortex sheet at any given time is composed of all the particles that have been shed through the separation point on the body contour. Therefore the vortex sheet is a streakline of the developing flow. Since the vortex sheets are material surfaces there is no flow across the vortex sheets and they must move with the local flow. Each vortex sheet is a free surface with both 
unknown position and strength. Therefore, two boundary conditions are required for its complete specification. The dynamic boundary condition (16) on each sheet is:

$$
\frac{\partial \gamma_{q}^{\mathrm{f}}}{\partial t}+\frac{\partial\left(v_{s} \gamma_{q}^{\mathrm{f}}\right)}{\partial s}+\gamma_{q}^{\mathrm{f}} \frac{v_{n}}{\rho}=0, \quad m=1,2, \ldots, M .
$$

The required kinematic boundary condition is,

$$
\frac{\mathrm{D}}{\mathrm{D} t} F_{m}(x, y, t)=\frac{\partial}{\partial t} F_{m}(x, y, t)+\vec{V} \cdot \nabla F_{m}(x, y, t)=0, \quad m=1,2, \ldots, M .
$$

Here $F_{m}(x, y, t)=0$ is the surface equation of the $m$ th vortex sheet. Equation (18) governs the rate at which the surface moves normal to itself. The rate of extension of the vortex sheet is determined by the local tangential velocity at its downstream end.

\subsection{Boundary conditions on the body}

The boundary condition to be satisfied on the body contour $C_{\mathrm{b}}$ is (4). This can be expressed as

$$
\begin{aligned}
& \vec{V} \cdot \hat{n}=0 \quad \text { on } C_{\mathrm{b}}, \\
& \vec{V} \cdot \hat{s}=0 \quad \text { on } C_{\mathrm{b}},
\end{aligned}
$$

where $\hat{n}$ and $\hat{s}$ are the contour unit normal and tangential vectors, respectively.

Here, consistent with the view that this model represents the infinite Reynolds number limit of a real flow, with the attached boundary layer being the bound vortex sheet, the material contour of the body is actually the inside contour of the bound vortex sheet. The no-slip condition of (20) must therefore be invoked upon approaching the contour from the inside. If this is done, the interior flow, corresponding then to a rigid body, is identically zero. The normal boundary condition, according to (19) is then identically satisfied on both sides of $C_{\mathrm{b}}$. This view of a potential flow as satisfying the complete body boundary conditions in the infinite Reynolds number limit is expounded by Lighthill [7] among others.

By requiring the tangential velocity to vanish according to (20) on approaching the contour from the inside, a Fredholm integral equation of the second kind in terms of the unknown vortex sheet strength is obtained from (11):

$$
-\frac{1}{2} \gamma_{p}^{\mathrm{b}}+\oint_{C_{\mathrm{b}}} \gamma_{q}^{\mathrm{b}} K_{p q} \mathrm{~d} s_{q}+\sum_{m=1}^{M} \int_{C_{m}} \gamma_{q}^{\mathrm{f}} K_{p q} \mathrm{~d} s_{q}+\vec{V}_{\infty} \cdot \hat{s}_{p}=0 .
$$

Here, counter-clockwise vorticity is considered positive. $\hat{s}_{p}$ is the unit tangential vector to the body contour at point $p$ and the positive direction for $\hat{s}$ is obtained by traversing the body contour in the counter-clockwise direction. $K_{p q}$ is the induced tangential velocity at point $p$ due to a unit point vortex located at the point $q$ and

$$
K_{p q}=\hat{s}_{p} \cdot \hat{k} \times \nabla_{p} G(p, q) .
$$




\section{Computational procedure}

The forward time-stepping of the initial value problem formulated as equations (17), (18), and (21) involves a recurring sequence of computations. In general, a new time step is executed with the application of the kinematic boundary condition, (18), to move the field vortex segments to a new position and to add a new segment at the downstream ends. This is followed by the application of the dynamic boundary condition, (17), to redistribute the vortex strength along the field vortex sheets. Next, the body contour vortex strength is resolved for the altered onset flow from the new configuration of field vortex sheets using (21). Finally, the time step is concluded with the computation of the field velocities over a fixed grid using (11), for the purpose of applying the kinematic and dynamic boundary conditions in the next time step. The five computation steps, or stages, constitute the streakline method. These stages in the streakline method are explained in more detail below.

\subsection{Computational stages}

\subsubsection{Initial condition}

The flow is started impulsively from rest. The body contour vortex strength $\gamma^{\mathrm{b}}$ corresponding to the attached potential flow solution provides (6), the starting point for the computation. This solution is computed from the boundary integral equation (21) with field vortex strengths $\gamma^{\mathrm{f}}$ set to zero. The body surface is divided into $N$ panels. Each panel is assumed to have a constant vortex strength. Thus, there are $N$ unknowns. Panel mid-points are chosen as control points. Equation (21) is discretized and applied at the $N$ control points on the body, giving the following $N$ simultaneous linear algebraic equations in $N$ unknowns,

$$
-\frac{1}{2} \gamma_{p}^{\mathrm{b}}+\sum_{\substack{q=1 \\ q \neq p}}^{N} \gamma_{q}^{\mathrm{b}} \int_{\Delta s_{q}} K_{p q} \mathrm{~d} s_{q}=-\vec{V}_{x} \cdot \hat{s}_{p}, \quad p=1,2, \ldots, N .
$$

These equations are inverted for $\gamma^{\mathrm{b}}$.

\subsubsection{Kinematic boundary condition}

Here, the kinematic boundary condition (18) is imposed along each of the vortex trailers present in the field. In the first time step, vortex segments are extended into the field from the sharp corners of the contour. Each such segment is an extension of the panel that is immediately upstream of the corner from which it is extended. The segment is extended tangentially from the parent panel with a strength equal to that of the parent panel. The length of each segment is equal to the product of the time step, $\Delta t$, and the tangential velocity $v_{s}$ along the parent panel. The tangential velocity $v_{s}$ is equal to $\gamma^{\mathrm{b}}$, the vortex strength of the parent panel.

Throughout the computation, the lengths of the individual segments of the field vortex sheets are kept constant. As a result, the vortex sheet can be viewed as a chain of rigid vortex segments which can only rotate at the joints. Consider a vortex trailer as shown in Fig. 1 after $m$ time steps. The trailer is attached to the body at the sharp corner $P$ and there are $i$ segments. In order to find the new position of the trailer, the calculated normal velocities in the previous time step are used to rotate the segments. The segments are rotated successively starting from the first segment. The segments of a vortex trailer are numbered successively 


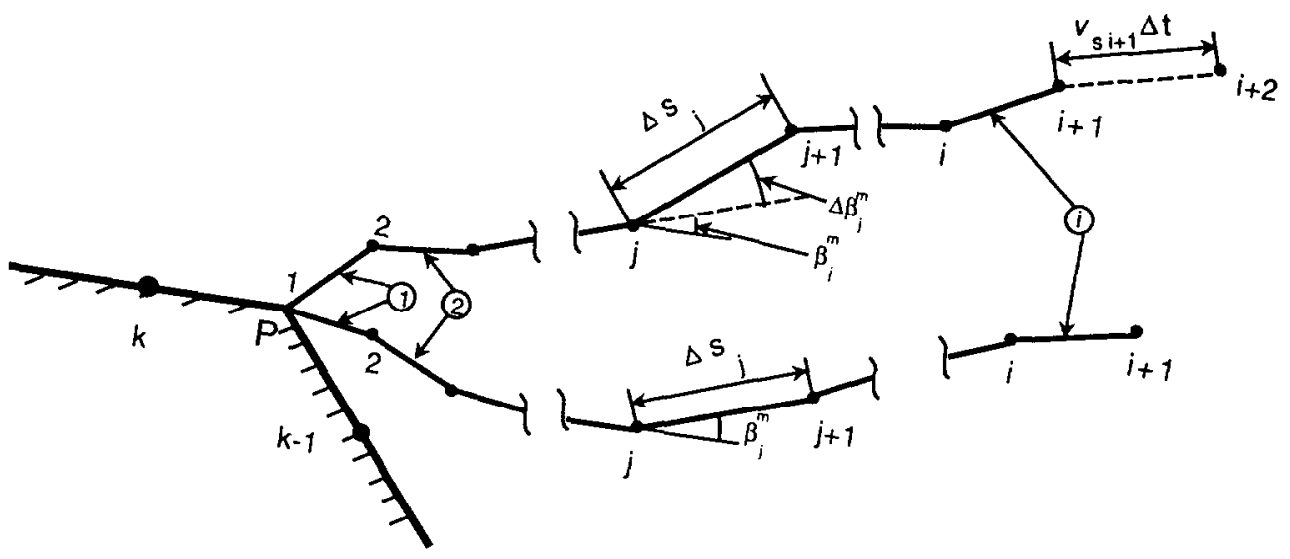

Fig. 1. Boundary conditions on a streakline.

starting from the one that is attached to the body. That is, the first point of the first segment, which is also a corner of the body, is fixed for all time steps:

$$
\begin{aligned}
& x_{1}^{0}=x_{1}^{1}=x_{1}^{2}=\cdots=x_{1}^{m}, \\
& y_{1}^{0}=y_{1}^{1}=y_{1}^{2}=\cdots=y_{1}^{m} .
\end{aligned}
$$

In the above and the following equations, superscripts denote the time steps and subscripts identify either a segment or its end point.

To find the new position of a particular trailer, segment 1 is rotated first about end point 1. The rotation $\Delta \beta_{1}$ determines the new position of point 2 relative to point 1 . Now, segment 2 is given the same amount of translation as that of point 2 . In addition, segment 2 is also given a rotation $\Delta \beta_{2}$. This finally fixes the position of end point 3. Similarly considering a general segment $j$, it is given the same amount of translation as the point $j$, and the rotation $\Delta \beta_{j}$ fixes the position of end point $j+1$ relative to $j$. The amount of rotation of each segment is given by the relative velocities normal to the segment at the two end points of the segment. The computational procedure is summarized in the following equations (refer to Fig. 1):

$$
\begin{aligned}
& \vec{N}_{j}^{m}=-\operatorname{Sin}\left(\beta_{j}^{m}\right) \hat{e}_{1}+\operatorname{Cos}\left(\beta_{j}^{m}\right) \hat{e}_{2}, \\
& V_{n j}^{m}=\vec{N}_{j}^{m} \cdot\left(V_{j+1}^{m}-V_{j}^{m}\right), \\
& \Delta \beta_{j}^{m}=V_{n j}^{m} \Delta t / \Delta s_{j}, \\
& x_{j+1}^{m+1}=x_{j}^{m+1}+\Delta s_{j} \operatorname{Cos}\left(\beta_{j}^{m}+\Delta \beta_{j}^{m}\right), \\
& y_{j+1}^{m+1}=y_{j}^{m+1}+\Delta s_{j} \operatorname{Sin}\left(\beta_{j}^{m}+\Delta \beta_{j}^{m}\right), \\
& \beta_{j}^{m+1}=\beta_{j}^{m}+\Delta \beta_{j}^{m} .
\end{aligned}
$$


Here $\vec{N}_{j}^{m}$ is the normal to the $j$ th segment in the $m$ th time step. $\beta_{j}^{m}$ is the angle made by the $j$ th segment with the $x$-axis in the $m$ th time step. $\Delta s_{j}$ is the length of the $j$ th segment which is between the end points $j$ and $j+1$. Unit vectors $\hat{e}_{1}$ and $\hat{e}_{2}$ are along $x$ and $y$ directions, respectively.

At the end of this normal movement, a new segment is added at the downstream end of the trailer. The length $\Delta s_{i+1}$ is equal to the product of the tangential velocity, $\left(v_{s}\right)_{i+1}$, at its last point and the time step $\Delta t$ as shown on Fig. 1. If the calculated length is below a minimum length, then $\Delta s_{i+1}$ is set to this minimum length. There are two reasons for this. One is to prevent the segments from rotating excessively for given normal velocitics (refer to (28)). The other reason for a minimum trailer segment length has to do with satisfying the dynamic boundary condition. Both these aspects are discussed in further detail in Section 3.2.

\subsubsection{Dynamic boundary condition}

The vorticity along the trailers is to be redistributed according to the vorticity transport equation (17). For this purpose, the tangential velocities calculated in stage 5 are used. The usual upwind differencing is employed to discretize the vorticity transport equation (17). The last term $\gamma v_{n} / \rho$ drops out for the following reason. $v_{n} / \rho$ represents the rate of stretching due to the normal movement of a circular-arc material segment while subtending the same angle at the center. In the current procedure, as described above, the individual segments are kept rigid and new segments are added at the end of the trailer to take into account stretching due to all the different mechanisms. Also since the kinematic boundary condition is applied first, i.e., the segment is moved normal to itself so that the normal velocity is zero in its new position,

$$
\begin{aligned}
& \frac{\partial \gamma}{\partial t}+\frac{\partial\left(v_{s} \gamma\right)}{\partial s}=0 \\
& \gamma_{j}^{m+1}=\gamma_{j}^{m}-\lambda^{+}\left[\left(v_{s} \gamma\right)_{j}^{m}-\left(v_{s} \gamma\right)_{j-1}^{m}\right]-\lambda^{-}\left[\left(v_{s} \gamma\right)_{j+1}^{m}-\left(v_{s} \gamma\right)_{j}^{m}\right],
\end{aligned}
$$

where

$$
\nu=v_{s} \Delta t / \Delta s
$$

and

$$
\begin{aligned}
& \lambda^{-}= \begin{cases}\Delta t / \Delta s, & \text { if } \nu_{j-1 / 2}<0, \\
0 & \text { otherwise, }\end{cases} \\
& \lambda^{+}= \begin{cases}\Delta t / \Delta s, & \text { if } \nu_{j+1 / 2}>0, \\
0 & \text { otherwise }\end{cases}
\end{aligned}
$$

The superscript $m$ denotes the time step number in the above equations. This is an upwind finite-difference scheme which automatically changes the direction of differencing depending on the convection velocity, $v_{s}$, along the trailers. In this scheme, the velocities are known at panel end points denoted as $j-\frac{1}{2}, j+\frac{1}{2}$, etc. The vortex strengths are given at panel midpoints denoted by $j-1, j, j+1$, etc. The number $\nu$ is evaluated by (33) at the panel end points. $\Delta s_{j-1 / 2}$ and $\Delta s_{j+1 / 2}$, to be used in the evaluation of $\nu$ at the end points, are taken as the averages $\frac{1}{2}\left(\Delta s_{j}{ }_{1}+\Delta s_{j}\right)$ and $\frac{1}{2}\left(\Delta s_{j}+\Delta s_{j+1}\right)$, respectively. The Courant-Friedrichs-Lewy (CFL) stability condition imposes a relationship between $\Delta t$ and $\Delta s$, and they cannot both be chosen arbitrarily, since $v_{s}$ is determined by the flow field. Special care has to be exercised at the first 
panel where the vortex trailer joins the body. Here the first panel of the vortex trailer can be fed vorticity from either or both of the body panels $k-1$ and $k$, depending on the velocity on the body contour. (See Fig. 1.) Equation (32) in general has to be applicd twice at the contour corner points.

After the vorticity redistribution is completed, the strengths of the newly added segments during this time step are checked against a minimum cutoff value. If the magnitudes of the vortex strengths of the added segments are below the cutoff value, they are discarded. Hence the vortex tube is extended into the field only as fast as it can be filled. This results in a more efficient computation. Further, empty tube segments successively added at the end tend to roll up. Therefore, the empty segments are discarded as they are generated.

\subsubsection{Body boundary condition}

At this stage of the computation, the velocity field around the body has changed. The boundary integral equation (21) is therefore re-solved. Here, the field vortex strengths $\gamma^{\mathrm{f}}$ are not zero; the discretized integral equation is obtained as,

$$
\begin{aligned}
-\frac{1}{2} \gamma_{p}^{\mathrm{b}}+\sum_{\substack{q=1 \\
q \neq p}}^{N} \gamma_{q}^{\mathrm{b}} \int_{\Delta s_{q}} K_{p q} \mathrm{~d} s_{q} \\
=-\sum_{m=1}^{M} \sum_{q=1}^{N_{m}} \gamma_{q}^{\mathrm{f}} K_{p q} \mathrm{~d} s_{q}-V_{\infty} \cdot \hat{s}_{p}, \quad p=1,2, \ldots, N .
\end{aligned}
$$

The first term on the right-hand side of (36) is the contribution of the shed vortex segments. $N_{m}$ is the number of vortex segments constituting the $m$ th field vortex sheet.

\subsubsection{Field velocity computation}

The field velocity is needed for applying the boundary conditions in the next time step. The total velocity due to the uniform stream and the bound and field vortex sheets is calculated at fixed grid points in space by (11). The equation is discretized into the form:

$$
\begin{aligned}
\vec{V}_{p}= & \vec{V}_{\infty}+\sum_{q=1}^{N} \gamma_{q}^{\mathrm{b}} \int_{\Delta s_{q}} \hat{k} \times \nabla_{p} G(p, q) \mathrm{d} s_{q} \\
& +\sum_{m=1}^{M} \sum_{q=1}^{N_{m}} \gamma_{q}^{\mathrm{f}} \int_{\Delta s_{q}} \hat{k} \times \nabla_{p} G(p, q) \mathrm{d} s_{q} .
\end{aligned}
$$

In using this formula, the induced velocity at any grid point falling within a specified radius from the midpoint of a vortex segment is approximated to be zero. This produces a spatial smoothing of the velocity field. The field velocity calculated at each grid point is saved for several successive time steps for the purpose of short time averaging to further smooth the velocity in time.

Velocity components at segment end points along each trailer are obtained by spatial interpolation of the short time averaged velocities at the fixed grid points. This produces yet another form of spatial smoothing of the velocity field. Spatial and temporal smoothing are further discussed in the following section. 
The calculated velocities are resolved along the tangential and normal directions to the trailer segments for the purpose of applying the kinematic and dynamic boundary conditions in the next time step.

Velocity due to vortex trailers and the uniform flow is calculated at the body control points to be used for solving the body vortex strengths from (36) of stage 4. Velocity at the corner points of the body is calculated as the average of the velocities at the two adjacent panel midpoints.

This completes one time step. The time step is now incremented and the computational procedure returns to stage 2 .

\subsection{Discussion}

In order to achieve steady flow in the near field of the body, it is obviously necessary to perform the sequence of computations outlined above for a large number of time steps. It is convenient to view the flow field as evolving on multiple time and length scales. The large time and length scales are associated with the gross features of the global flow, which are the characteristics of interest here. The small time and length scales, on the other hand, are associated with the instabilities and vortex sheet roll-up, which are not of interest in the present study, but which tend to limit computations of this type to relatively small time. The troublesome small scale features are eliminated here by filtering, or smoothing the flow field as it evolves in time and space.

Filtering or smoothing in time is accomplished by averaging the velocity field over a pre-selected number of successive time steps as given below:

$$
\tilde{\vec{V}}=\frac{1}{\delta t} \int_{t-\delta t}^{t} \vec{V} \mathrm{~d} t
$$

Here $\tilde{\vec{V}}$ refers to the time averaged field velocity. The integral in the above equation is replaced by a summation over a number of time steps $n_{\text {avg }}$, where $\delta t=n_{\text {avg }} \Delta t$, giving

$$
\tilde{\vec{V}}=\frac{1}{\delta t} \sum_{k=m-n_{\text {avg }}}^{m} \vec{V}^{k} \Delta t=\frac{1}{n_{\text {avg }}} \sum_{k=m-n_{\text {avg }}}^{m} \vec{V}^{k}
$$

In satisfying the kinematic (18) and dynamic (17) boundary conditions in stages 2 and 3 above, velocities are needed only along the field vortex sheets. However, the time averaging must be performed at fixed points in space, rather than along the field vortex sheets, which move with time. For this reason, field velocity calculations are performed on a fixed grid. A rectangular grid is generated in the field. A triangular grid is generated in the local region of the body for accuracy in matching the general body contour. Refer, for example, to Figs. 2 and 3 . The triangular mesh is such that the vertices of the triangles that fall on the body contour are made to coincide with the end points of the body panels.

Smoothing of the flow field in space is accomplished primarily by spatial interpolation of the field velocities. The induced velocities due to the trailers are calculated at the fixed grid points in the field, and then interpolated back to the trailers in satisfying the boundary conditions. In maintaining a field grid with spacing much greater than the length of the longest field vortex segment, a spatially smooth velocity field is naturally achieved at the trailers, except in the case 
where a vortex segment falls in the immediate vicinity of a grid point. In such a case, the limiting far field representation of the vorticity shear layer as a vortex sheet is not appropriate. The alternative limiting near field model is used instead. This is an infinitc uniform vorticity field surrounding the grid point, for which the induced velocity is identically zero. The decision on which of the two limiting cases to use in any particular situation is decided on the basis of a pre-selected "cutoff radius". The maintenance of a large cutoff radius further smoothes the velocity field by removing large velocities that occur at points in the vicinity of the vortex segments, due to the thin layer approximation of the field vorticity. This "cutoff radius" is denoted as $r_{\delta}$. A cutoff radius of 3.0 means that the induced velocity at a point due to a vortex segment of length $\Delta s$ is zero if the point lies in a circle of radius $3.0 \Delta s$ centered at the midpoint of the segment. This removes the artifice of infinite velocities in the neighborhood of the singularities used to represent the flow. It is justified by the argument that in the local near field, the vorticity is distributed rather than compressed into an infinitesimally thin sheet.

Vortex sheet roll-up is further suppressed by selectively adding new trailer end segments, as cited earlier. Recall that a new segment is added at the downstream ends of the vortex sheets at every time step in satisfying the kinematic boundary condition. The newly added segments have zero vorticity. Vorticity flows into them during the dynamic boundary condition stage as dictated by (32). After satisfying the dynamic boundary condition, the magnitude of the vortex strengths are checked against a minimum value. If the vortex strength of a particular new end segment is smaller than this minimum value, then the newly added segment is discarded. The vorticity that is present in this discarded segments is assumed to be lost to the field and dissipated there. If new segments are indiscriminately added during every time step, then there will be many end segments of very small vorticity level. This leads to a situation where the rate of extension of the vortex sheet is higher than the rate of change of sheet strength. This further leads to roll-up of the sheet. By preventing the unchecked extension of the sheet, the roll-up of the "empty" vortex sheet ends is eliminated, which is the desired goal.

\section{Results}

The streakline method has been applied to calculate the free-streamline flow about the following contours: a knife edge, a rectangular box, a circle represented by a polygon, and a circular arc represented by a polygon. Results for the rectangular box are not presented here and the reader is referred to [3]. For all these shapes the $x$-axis is in the horizontal direction and the $y$-axis in the vertical direction. The dimensions, the orientation with respect to the uniform flow and the near field grid are shown in Figs. 2 and 3. These problems are non-dimensionalized by the uniform free-stream velocity and a characteristic body dimension. In the cases of the knife edge and the rectangular box, the half height is chosen as unity. The radius is set to one in the two circle cases. In all of the calculations, the flow is assumed to be symmetric about the horizontal axis and the condition of zero circulation is identically satisfied for all time because of the prevailing symmetry.

A study was undertaken during the course of the calculations to examine the sensitivity to the different parameters that have to be selected a priori. The parametric study is presented first, before presenting the computed results for the four cases identified above. 


\subsection{Parametric study}

Being the simplest shape, the knife edge of Fig. 2 was chosen for the parametric study. The dimensions of the knife edge are: the half height (above the $x$-axis) is taken as 1.0 and the width as 0.2 . The edge angle is 45 degrees, as indicated on Fig. 2. The half body contour is divided into 20 panels of equal length.

For this case, there are two sharp corncrs at which the flow can separate from the body. The flow is allowed to separate from only the forward of the two sharp corners, which is numbered point 11 on Fig. 2. Although vortex sheets would be shed initially from both the corners, only the one shed from the forward corner would persist at large values of time. Using only the forward separation point was found to satisfactorily serve the purpose of the parametric study.

Table 1 identifies the various parameters involved in the calculation.

The time step size $\Delta t$ can actually be viewed as an independent variable. This being an explicit method in time, the time step size $\Delta t$ must be small in order to achieve computational accuracy. The time step size chosen for these calculations is 0.005 . Once the time step size is selected, the minimum length of the newly added trailer segments is automatically limited. The time step size and minimum segment length are selected such that together they satisfy the required dynamic (CFL) and kinematic stability conditions as discussed before. The smallest segment $\Delta s_{\min }$ that can be added at the downstream ends of the vortex sheets is taken as 0.015 . As discussed earlier, the vortex strength of the newly added segments is checked against $\gamma_{\min }$ after applying the dynamic boundary condition. If the strength of the newly added segment is below $\gamma_{\text {minin }}$, then this segment is discarded. This is merely to prevent accumulation of almost

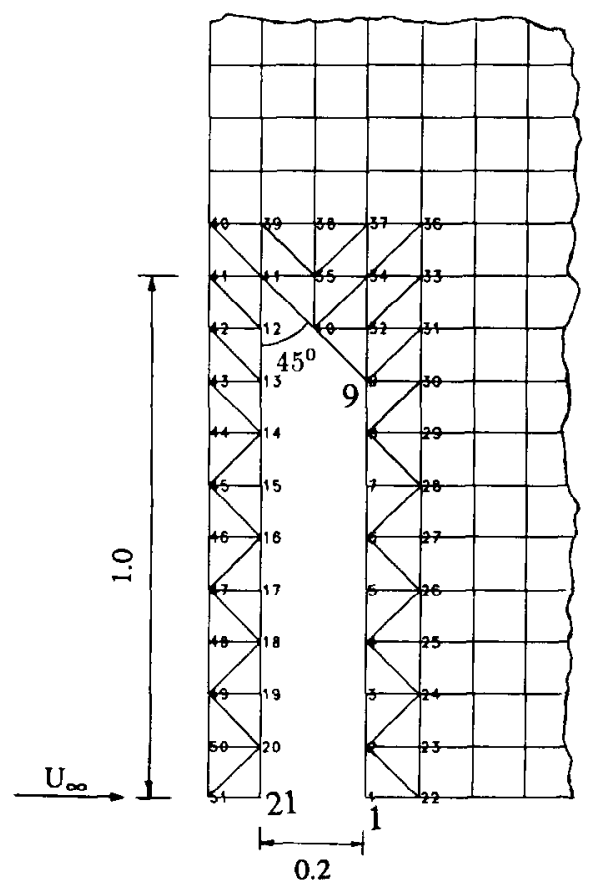

Fig. 2. Near field grid: knife edge. 
(a)

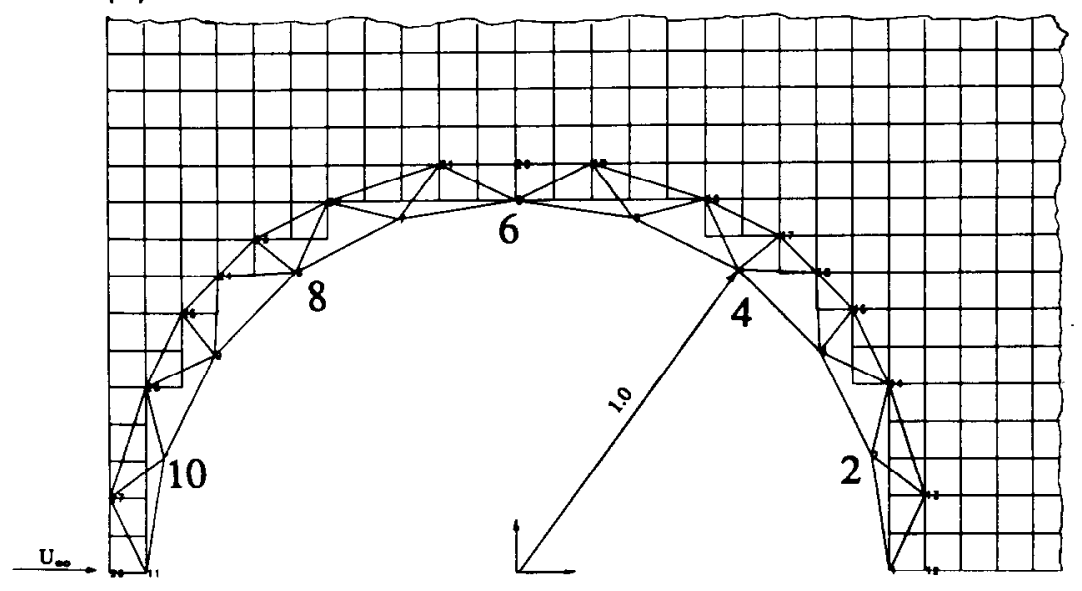

(b)

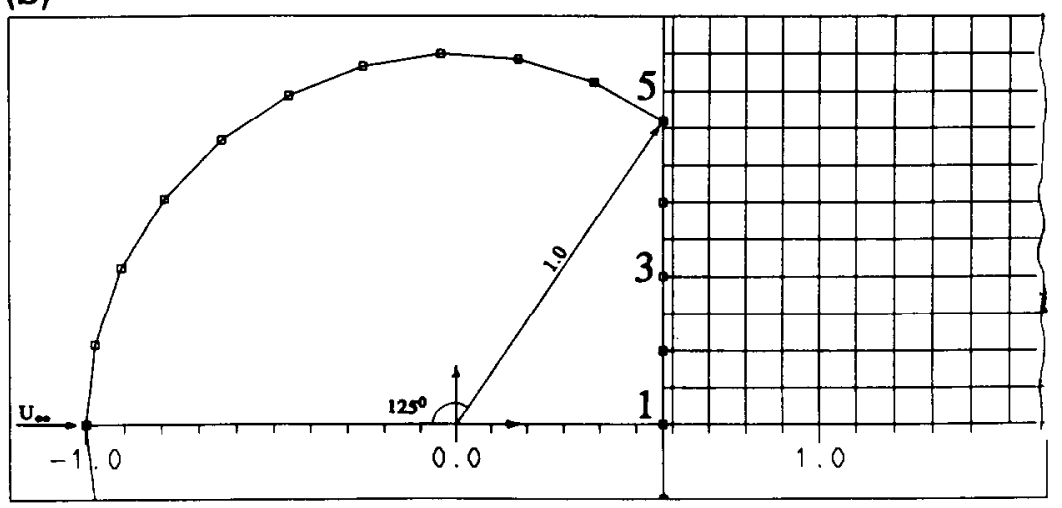

Fig. 3. Near field grid: (a) circle, (b) circular arc.

empty segments as the downstream ends of the vortex trailers, as previously discussed. For this study, $\gamma_{\min }$ is selected as 0.01 .

The remaining three parameters of Table 1, i.e., $r_{\delta}, n_{\text {avg }}$, and $h$, were varied systematically in computations with the knife edge. The results are summarized in Fig. 4. The developing vortex sheets are shown in Fig. 4, with the number of time- steps corresponding to each configuration

Table 1

Computation parameters

\begin{tabular}{ll}
\hline Symbol & Parameter \\
\hline$\Delta t$ & Time step size \\
$(\Delta s)_{\min }$ & Minimum segment length to be added \\
$\gamma_{\min }$ & Value of $\gamma$ below which the newly added end segment is discarded \\
$r_{\delta}$ & Cutoff radius in the velocity calculation \\
$n_{\text {avg }}$ & Number of time steps over which the field velocity is averaged \\
$h$ & Rectangular grid size \\
\hline
\end{tabular}




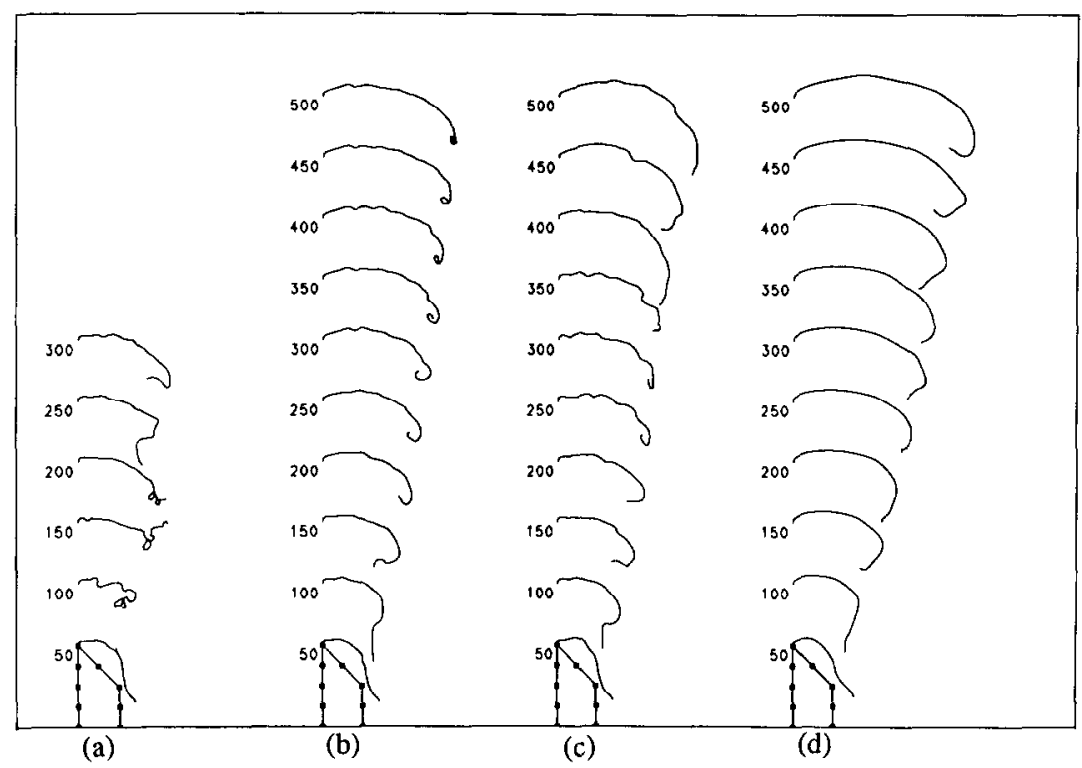

Fig. 4. Parametric study.

identified. Four test cases are performed for flow past the knife edge. In all these cases, $\Delta t$ and $\Delta s_{\min }$ are held fixed at the above values.

In all of the cases that follow, the triangular grid in the immediate vicinity of the knife edge was not changed, only the surrounding rectangular grid size was varied. This is considered to be permissible on the basis of the observation that computational instabilities, if they develop, always develop well after the flow has commenced, and after the trailers have convected well into the rectangular grid region. The flow always seems to start out cleanly almost regardless of the parameter values. This can be observed from Fig. 4. At 50 time steps, all the four cases look very much alike. Also observe from Fig. 2 that the extent of the triangular grid around the body is very limited.

Case I: Here, the three parameters are chosen as follows: the cutoff radius $r_{\delta}=0.01$, field velocity is not averaged, i.e., $n_{\text {avg }}=1$, and the size of the rectangular grid, outside the triangular grid, is chosen as 0.025 , i.e., $h=0.025$. Observe that the mesh size 0.025 is of the same order of magnitude as the minimum segment length of 0.015 . The cutoff radius $r_{\delta}$ is only $1 \%$ of the segment length.

Referring to Case I on Fig. 4(a), it is clear that the calculations become unstable very quickly. The breakdown commences even before 100 time steps. Even though the streakline appears to have become smooth after time step 250, the calculations could not be carried out beyond 300 time steps, where a numerical breakdown occurred.

Case II: This case is chosen to demonstrate the effect of variation of the cutoff radius, $r_{\delta}$. The cutoff radius is increased from 0.01 to 0.1 . All the other parameters are kept the same as in Case I. The parameters are listed again for reference as: $r_{\delta}=0.1, n_{\text {avg }}=1$, and $h=0.025$. The mesh size is the same as in the previous case and field velocities are not averaged. The cutoff 
radius now is ten times greater than that in the previous case. This implies greater spatial smoothing, as the field velocity gradients in the vicinity of a vortex segment are reduced.

From Fig. 4(b) it is clear that the calculation in Case II is more stable than in Case I. However, small kinks can be observed as the calculations progress. Furthermore, starting from time step 250, a definite roll-up can be observed. By time step 500, the vortex sheet is rolled up into a very tight spiral at its end and the computations were stopped. By comparing Cases I and II, it is obvious that the cutoff radius plays a major role in smoothing the flow field. However, the vortex sheet shows a tendency to roll up at its end.

Case III: Here, the effect of time averaging the velocity field is demonstrated. The cutoff radius and the mesh size are taken 0.01 and 0.025 respectively. These values are the same as in Case I. The field velocities are stored over five successive time steps and are averaged. i.e, $n_{\text {avg }}=5$. From Fig. 4(c), up to time step 250, Cases II and III are quite similar. Subsequently the roll-up of the vortex sheet in Case II is replaced in Case III by an increasing general irregularity. The irregularity reflects the small (Case I) cutoff radius. The roll-up is avoided in Case III by the time averaging. Mechanically, the time averaging produces a phase shift between the self-induced velocity at the trailer end, and the position of the trailer end. The trailer end essentially "out-runs", downstream, the self-induced velocity tending to roll it up.

Case IV: In this case, the grid size is varied. The grid size is increased from 0.025 to 0.1 . Now, the grid size is 6.7 times the minimum vortex segment length. As discussed earlier, the sheet model of the vorticity shear layer is a far field approximation. Therefore, the larger the grid size in relation to the segment length, the better the velocity calculation as a far field approximation. Grid size is obviously an additional spatial smoothing parameter. The larger the grid size, the farther away are the vortex segments and the smoother the velocity field. The grid size of course could be made excessive. Then the required velocities along the trailers are interpolated from too large a region, not reflecting well enough the local velocity field characteristics. It can be seen from Fig. 4(d) that the Case IV sheet is quite smooth, where at the same time possessing the same global character of the sheets of Cases II and III. Observe that increasing the grid size also has some effect in suppressing the sheet roll-up.

Therefore with proper selection of the respective parameters, two-dimensional vortical flows can be time-stepped to average steady state with the streakline method, without serious difficulty imposed by the persistent small scale flow structure along the way.

We now return to the subject computations with the flow cases of Figs. 2 and 3. The values of the cutoff radius employed in each of these cases are much larger than the values of 0.1 and 0.01 selected for the parametric study. In retrospect, a much smaller $r_{\delta}$ could probably have been used. The larger values were arrived at by consideration of the order of magnitudes of the induced velocities. The magnitude of the velocity at a distance $r_{\delta} \Delta s$ induced by a point vortex situated at the midpoint of a vortex segment of length $\Delta s$ and strength $\gamma$ is

$$
q=\frac{\gamma \Delta s}{r_{\delta} \Delta s}=\frac{\gamma}{r_{\delta}} .
$$

For the cases under consideration, $\gamma$ is initially between 1.5 to 3.0. As time progresses, the strength decreases toward 1.0. Hence taking the order of magnitude of $\gamma$ as 1 ,

$$
q \sim \frac{1}{r_{\delta}} .
$$




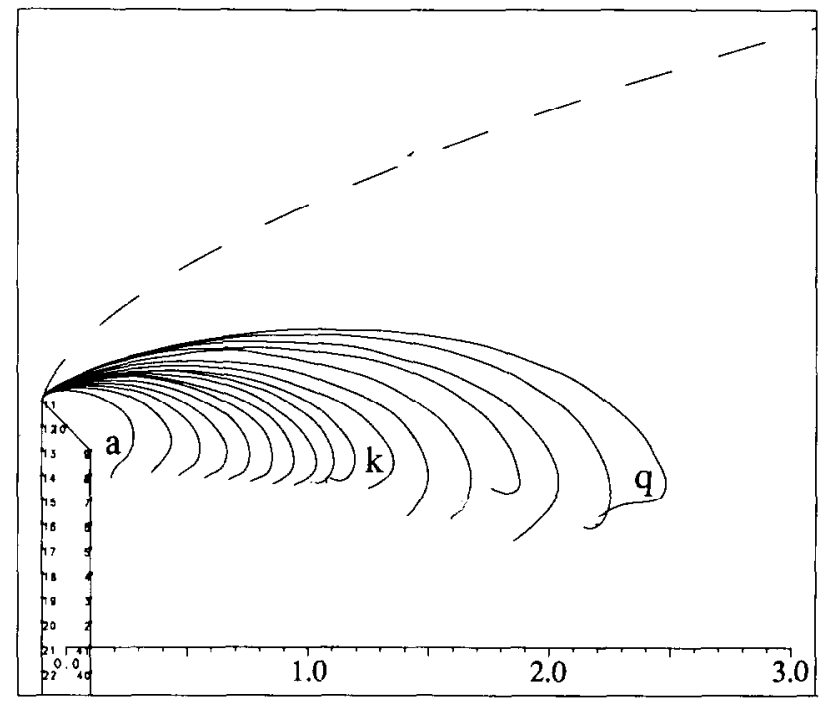

Fig. 5. Flow development past the knife edge with one separation point. The letters a, $k$, and q denote the time steps 120,1220 , and 2420 , respectively. The streaklines are shown at an interval of 100 time steps between a and $\mathrm{k}$ and at an interval of 200 time steps between $\mathrm{k}$ and $\mathrm{q}$.

The onset stream velocity is of magnitude 1.0. If a cutoff radius $r_{\delta}=0.1$ is selected, the magnitude of the induced velocity is then 10 times that of the onset velocity. Since this can lead to numerical instabilities, $r_{\delta}$ was selected such that the induced velocity is only a fraction of the onset velocity, although much smaller $r_{\delta}$ could have apparently been used.

The results obtained from the streakline computations for the four different shapes cited are presented in the following sections.

\subsection{Knife edge with one separation point}

Here the analysis of the knife edge with one separation point (Fig. 2) is continued with a finally selected parameter set. Referring to Table 1 , the following parameter set used is: $\Delta t=0.005,(\Delta s)_{\min }=0.015, \gamma_{\min }=0.01, r_{\delta}=3.0, n_{\text {avg }}=5$, and $h=0.1$. The time-stepping was carried to 2420 non-dimensional time steps.

The streakline configuration versus increasing time steps is shown in Fig. 5. The dashed line is the well-known Kirchhoff free-streamline solution for stationary flow past a normal flat plate situated at the forward face of the knife edge. The analytical solution is given by Lamb [5]. The characteristics of the analytical solution are as follows. The flow separates at the tip of the flat plate and a free streamline proceeds downstream toward infinity. A cavity, open at infinity, is formed below the free streamline and behind the body. Within this cavity there is no flow. The velocity on the free streamline is equal to that of the free stream at infinity. Hence the free streamline is also a vortex sheet with vortex strength equal to -1.0. As the time-stepping proceeds to some large values of time, the calculated solution for the knife edge should match the Kirchhoff free-streamline solution for the flat plate, since at steady state the shape of the body below the free streamline is inconsequential because of stagnant flow condition that exists within the cavity region. 
The solid lines shown in Fig. 5 are the positions of the vortex sheet at the different time steps indicated. As can be clearly seen, the shed vortex sheet is moving toward the analytical solution, but very slowly. Of course, infinite time would be required in order for the calculated solution to match the analytical solution exactly but a better near field comparison than that shown in Fig. 5 would be expected within some large but finite number of time steps.

The computations were not continued beyond the 2420 step maximum of Fig. 5 largely because of limitations of the Apollo mini computer system on which the programs were developed. The major limitation was the computing time and storage space for the field grid velocities. The complete computation of Fig. 5 with more than 2420 time steps, required approximately 72 hours of CPU time of an Apollo DN 3000 mini computer. The field grid spanned two plate breadths in the $x$ direction and one-and-a-half plate breadths in the $y$ direction, and the $n=2420$ trailer was composed of 216 segments. For the more conventional Lagrangian computations involving, say, $N$ point vortices at some time step, the number of induced velocity computations is $N^{2}$, since velocity is computed at each point vortex. With velocity computation on the fixed grid, and interpolating back to the vortex segments, the number of computations is ncccssarily larger. For $N$ vortex segments, and with grid dimensions, say, $M \times K$, the number of computations is of the order $N \times M \times K$. With a grid covering the entire extent of the trailers downstream and above the axis, as was employed in the examples demonstrated here, this computation could become on the order of $N^{3}$, as the grid must expand to contain the growing trailers. An adaptive grid generation scheme is the obvious recourse here. By maintaining an active field grid only large enough to surround the trailers for $n_{\text {avg }}$ successive time steps, the computations should be reducible to nearly order $N^{2}$. However, the achievement of near field steady-state solutions is not considered necessary to the main objective of the work, which was to investigate ideal flow separation point selection on general polygonal contours, as discussed in the introduction. It will be demonstrated in several of the following examples that the separation point selection process is clearly completed within a number of time steps of the order of the maximum of Fig. 5. The question of convergence to steady Kirchhoff flow is important however, as that is the solution to which the computation must extrapolate if it is to be judged accurate for any purpose. This interest in the steady flow limit was the primary motivation for selecting the final example presented in this section. By the analytical theory, the separation streamline of the 124.21 degree circular arc forms a separation cavity which closes in a cusp on the $x$-axis at infinity downstream. Thus the domain of the steady streamlines in that case are of much less extent than for any of the other cases, whose cavities are open, with infinite width, at infinity. Here, as will be shown, a satisfactory level of convergence toward the cusped analytical solution in the near field is considered to have been achieved.

The velocity field corresponding to the streakline position at time step 2421 is plotted in Fig. 6 as a vector field. Arrows indicate the direction of the velocity and the lengths of the arrows indicate its magnitude. The tail of the arrow is plotted at the point of calculation. An arrowhead with zcro length indicates that the velocity at that point is zero, or very close to zero. By observing the plot, it is obvious that the evolving solution is developing the essential characteristics of the Kirchhoff analytical solution for steady free-streamline flow. The velocities behind the body and below the free streamline are quite small and a jump in tangential velocity is apparent across the streakline. The enlarging cavity under the streakline is fed by the local reversed flow through the channel under the streakline end. This flow circulates around 


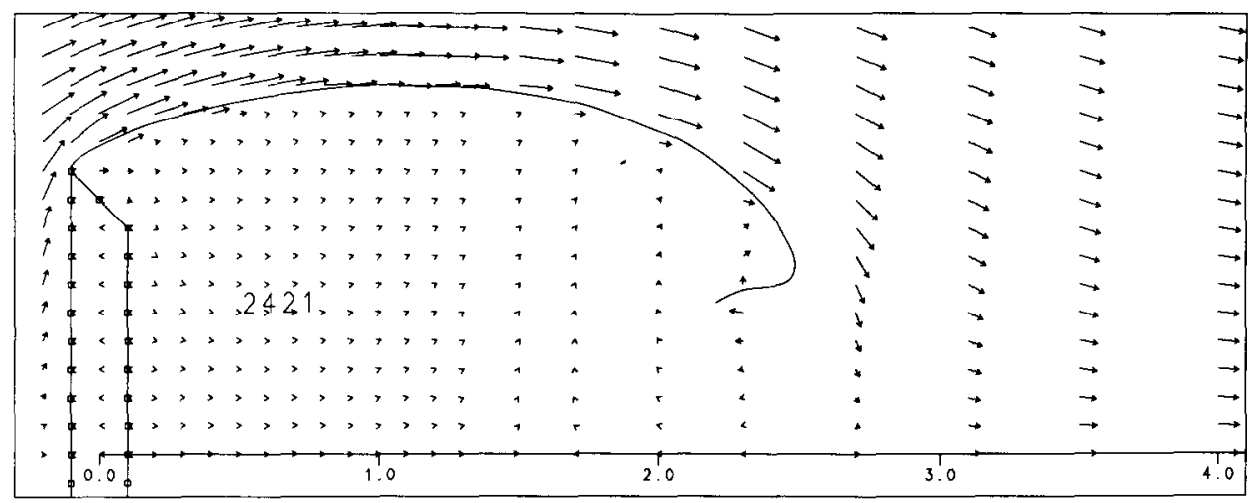

Fig. 6. Velocity vector field at time step 2421 for the flow past the knife edge with one separation point.

and upward inside the tip to provide the normal velocity for the streakline cavity growth downstream.

The vortex strength along the sheet of Fig. 6 is plotted on Fig. 7 versus distance along the sheet measured from the origin, at the separation point, for the different time steps. The dashed line is the line of magnitude -1.0 , which is the required vortex sheet strength for the ultimate steady flow. The vortex strength of the evolving sheet has decreased in magnitude continuously from -2.0 to nearly -1.5 . Note also that the magnitude is nearly constant over the entire length, except very near the ends where it decreases sharply. This is because time is required to fill the newly added segments with vorticity by convection from upstream; the streak tube lengthens at a rate necessary to contain the flowing vorticity.

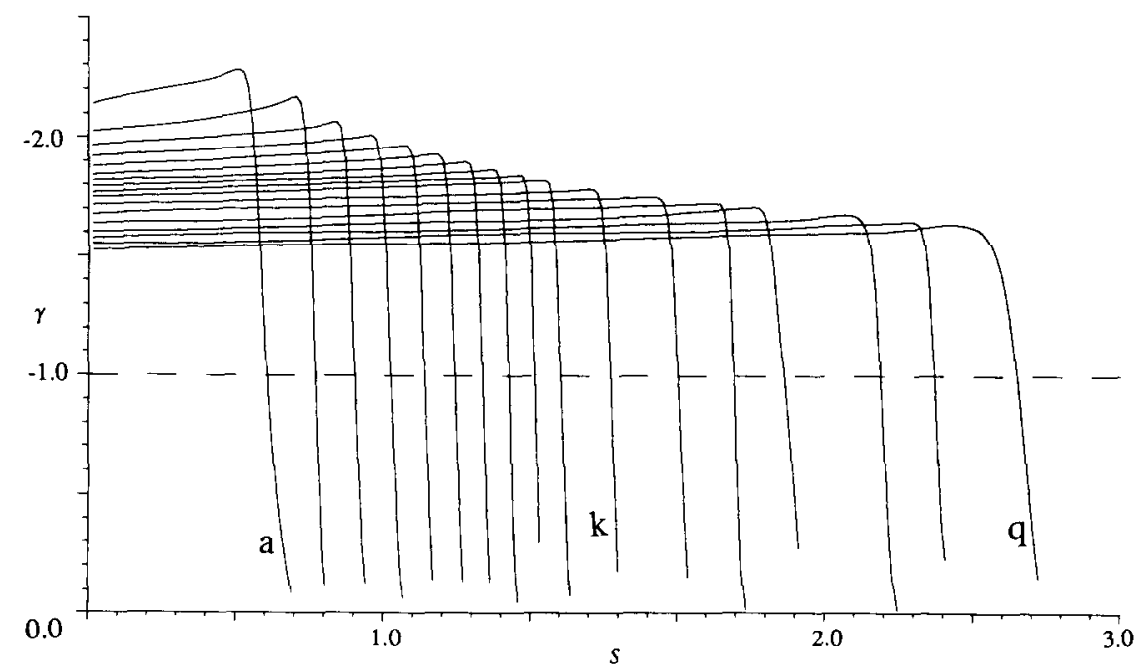

Fig. 7. Vortex strength along the streaklines at different times for the knife edge with one separation point. The letters a, $k$, and q denote the time steps 120,1220 , and 2420 , respectively. The streaklines are shown at an interval of 100 time steps between a and $\mathrm{k}$ and at an interval of 200 time steps between $\mathrm{k}$ and $\mathrm{q}$. 
The vortex strength is converging toward the value of -1.0 , but indeed slowly. The vortex strength $\gamma$ at the knife edge tip $(s=0)$, versus the natural logarithm of the time, is plotted as a function of time (not shown) to see the rate at which the vortex strength is approaching its asymptotic value. The plot of vortex strength versus time is nearly a straight line with negative slope, indicating that the decay rate of the vortex strength is exponential. The slope is -0.1914 and the $y$-intercept is 2.042 . From this, the time needed to reach a steady-state strength of -1.0 is estimated to be 231.4 non-dimensional time units. At a time step size of 0.05 , this implies 46,278 time steps from the start-up to achieve the steady-state vortex strength of -1.0 .

Another calculation was carried out to study the effect of shedding vorticity from the two sharp corners, 9 and 11, shown in Fig. 2. Vortex sheets are shed simultaneously from both the corner points 9 and 11 of the knife edge of Fig. 2. The most interesting feature of this calculation is that the vortex sheet shed from the downstream corner "dies" slowly with time. The strength of the downstream vortex sheet at time step 2420 was -0.1 compared to -1.5 of the leading sheet. "Death" of the vortex sheets occurs for two reasons:

(a) As the flow develops, a vortex tube may be fed positive vorticity from one body panel and negative vorticity from the adjacent panel, leading to vorticity cancellation. Here, redistribution along the sheet obviously results in a net weakening of the sheet vortex strength.

(b) As previously described, a new segment is added at the end of the vortex trailer only if, after redistribution of the vorticity, its strength is higher than a cutoff value. In steps where a new end segment is not added, a small amount of vorticity "dribbles" out of the tube ends into the ficld, where it is assumed to be dissipated.

The latter process represents a numerical artifice, whereas the former is rigorously justified. Of the two possible separation points on the knife edge, the calculation method picks the upstream corner, which is obviously the physically correct separation point.

Similar calculations are carried out for flow past a rectangular box. Vorticity is shed from the two top corners and just as in the case of a knife edge, the vortex sheet shed from the downstream corner dies down and the one from the upstream corner prevails for longer times.

\subsection{Circle}

The semi-circular cylindrical case of Fig. 3(a) represents the modelling of a smooth contour by a polygonal contour with a multiplicity of "corners" serving as streakline separation points.

An analytical solution procedure for stationary free-streamline flow past curved obstacles was first formulated by Levi-Civita [6] in 1907. In the 1920 s and early 1930s, the problem was further studied by Brodetsky [2] and in greater detail by Schmieden [10-13]. The Levi-Civita solution method and solutions for several cases are well documented by Birkhoff [1]. The particular case of interest here is the circular cylinder. The analytical free-streamline solution for symmetric flow past an obstacle is indeterminate to one degree. In order for the solution to be unique, the separation point must be specified from the set of all the feasible separation points. For the particular case of the circle, the flow can separate from the body anywhere between angles of 55.04 and 124.21 degrees, where the angle is measured from the forward stagnation point. The only streamline that has a continuously concave curvature towards the $x$-axis is the one that separates at 55.04 degrees, which is the forward extremity of the feasible 
solution range. The streamline that separates at the after extremity at 124.21 degrees has continuously convex curvature towards the $x$-axis and ends in a cusp on the $x$-axis at infinity. This cusped solution forms a closed separation cavity with zero drag. All other streamlines separating between these two extremes are initially convex towards the $x$-axis, but have an inflection point further downstream resulting in an open cavity with concave curvature at infinity. It is therefore not at all clear, beforehand, how the streakline method will perform with regard to this indeterminacy in the steady analytical solution for the circle.

Referring to Fig. 3(a), a unit radius is used for the circle represented by an inscribed polygon. The semi-circle contour is divided into ten panels of equal length. The computation parameters are: $\Delta t=0.005,(\Delta s)_{\min }=0.025, \gamma_{\min }=0.05, r_{\delta}=5.0$, and $h=0.1$. Special numerical problems arise in applying the streakline method to a polygonal contour with many corners. Ideally, vortex sheets are shed from all the corners. There is a tendency, however, for the closely spaced streaklines to intersect each other in the initial stages of the flow development. This can apparently be avoided by the use of a very small time step until the trailer configuration spreads sufficiently with time. For the present calculations, however, the time step of 0.005 was used throughout, but streaklines were shed from alternate corners on the contour to avoid the numerical "clashing" problem. The vortex sheets are shed from corners 2 , $4,6,8$, and 10 counting from the rear stagnation point in the anti-clockwise direction as shown in Fig. 3(a). Due to the close proximity of several vortex sheets to a given grid point, high velocities may be induced at this grid point. For this reason the cutoff radius, $r_{\delta}$, is chosen to be higher than the value of 3.0 used in the previous cases. Further, to avoid excessive rotation of the individual segments due to higher induced velocities, $\Delta s$ is also chosen to be higher than the previous value of 0.015 .

In the preceding knife edge and step cases it was clear that the boundary layer would separate at both corner points in proceeding forward in time from the initial attached potential flow. For the case of the circular polygon, however, the boundary layer does not necessarily separate at all of the corner points, at all times. Specifically, some of the corners on the forward face would not be expected to shed streaklines, but which ones do and which ones do not is not known a priori. In fact, shedding from the face points may change over the course of the flow development as induced velocities change over the polygon face. In the interest of dealing with this complexity so as not to bias the computation, streaklines are given the opportunity to shed from all of the chosen shedding points over the entire contour at every time step. Generally, the streakline is shed from a given corner for several successive time steps after start-up. If none of the several segment end points of the new streakline have moved farther than $0.2 \%$ of the circle radius into the field, then the streakline is eliminated, and restarted from the point as a new streakline in the next time step.

A radius criterion of $0.09 \%$ is used as a standoff limit in the kinematic boundary condition for field trailers migrating back toward the contour. If at any time in the course of the computation a trailer segment moves inside the $0.09 \%$ limit, the segment is moved to a radius of 1.002 , with the positions of the other trailer segments adjusted according to the kinematic boundary condition algorithm as given in Section 3.1.2.

Figure 8 shows the vortex sheet configurations at four time steps. The shedding is allowed to occur from the alternate corners, numbered 2, 4, 6, 8, and 10.

By time step 820 , shown in Fig. 8(b), the boundary layer is clearly separating from 54 degrees (corner 8 ), as the vortex sheets from the corners $2,4,6$, and 8 have cleared the contour and are 

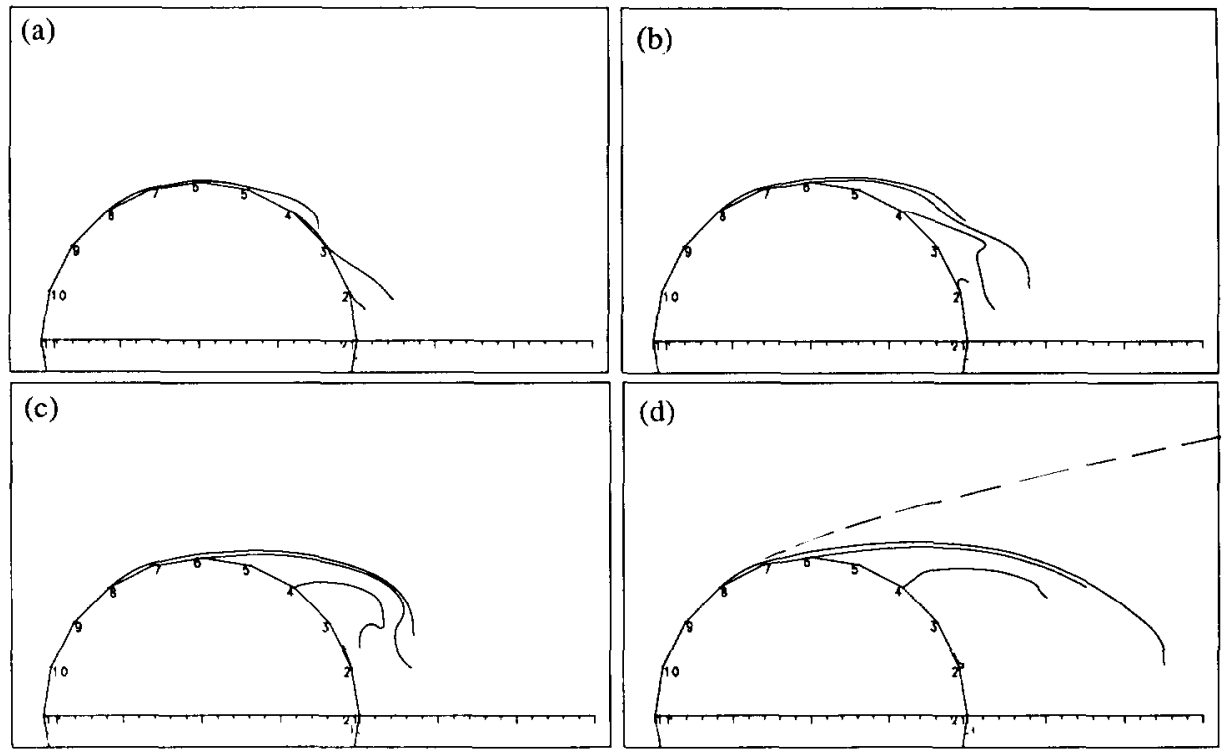

Fig. 8. Flow development past a circle. (a) At time step 420, (b) at time step 820, (c) at time step 1020, and (d) at time step 2420 .

well established as field trailers. The vortex sheet from corner 10 , which is being repeatedly extended into the flow along the tangent to the forwardmost contour panel, is being repeatedly absorbed back into the bound vortex sheet by the criterion previously described. This occurs because the net normal convection in the vicinity of the forward stagnation point is inward, being dominated by the axial uniform stream velocity component.

Note the reversed flow developing at the rear of the cylinder, as evidenced by the continuous upturning of the corner 2 trailer in Fig. 8 at time step 1020. Figure 8(c) shows that this reversed flow is fully established by this time step, as the corner 2 trailer has rotated into a fixed attitude coincident with the tangent up the back of the cylinder. By time step 1620, not shown, the two outer vortex sheets from corners 6 and 8 have begun to null the interior flow. The strengths of the two outer trailers at $n=1620$ are -0.28 and -1.2 respectively, on average, over their lengths versus -0.15 and +0.09 for corner 4 and 2 trailers, respectively. By time step 2420 , in Fig. 8(d), the growth in length of the three inside trailers has largely ceased, and their strengths are diminishing relative to the outer sheet, which would become the free streamline upon reaching the steady flow state. The velocity field at time step 2521 is plotted in Fig. 9.

The dashed line superimposed on Fig. $8(\mathrm{~d})$ is the analytical solution for the circle corresponding to the separation angle of 55.04 degrees. This is the forwardmost of the infinitely many possible separation points between 55.04 and 124.21 degrees according to the classical theory [1].

Of course, the computed result of Fig. 8 is fortuitous to the degree of latitude allowed in the selection of the separation point position on the polygonal contour. With the contour represented by ten panels, with shedding from alternate panel corners, the only separation points available for the streakline method to select from were 18, 54, 90, 126, and 162 degrees. Three of the five possibilities do however lie essentially within the range of the indeterminacy, 55.04 


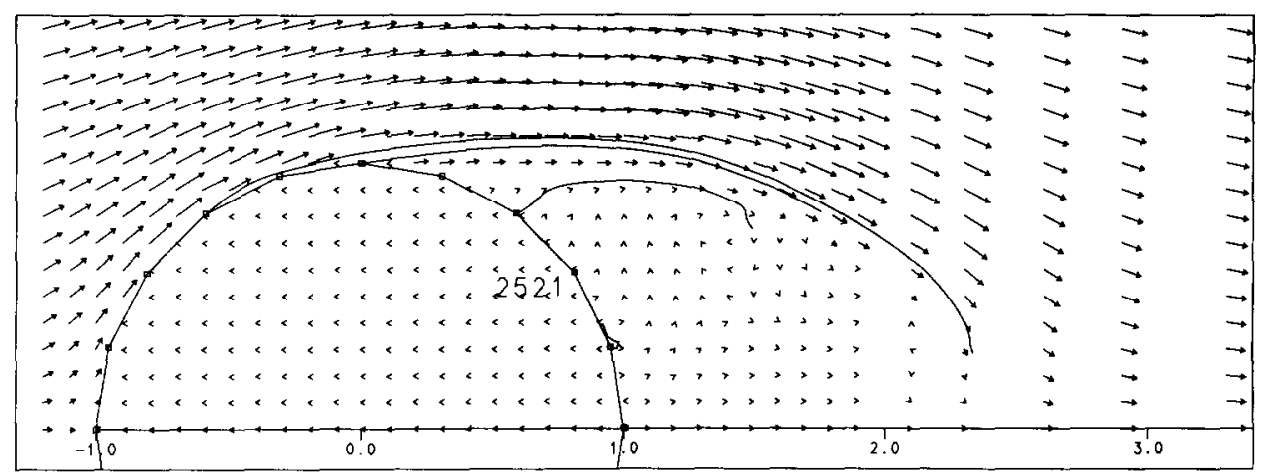

Fig. 9. Velocity vector field at time step 2521 for flow past the circle.

to 124.21 degrees, of the analytic solution for the smooth circle. Further study would be required in order to clarify the implications of this result.

\subsection{Circular arc}

On reviewing the analytic theory in connection with the preceding circular polygon example, it was noted that the two extremes of the indeterminate solution are a maximum drag solution corresponding to a continuously convex streamline separating at 55.04 degrees, and a zero drag solution with a continuously concave (towards $x$-axis) streamline separating at 124.21 degrees. The concave streamline ends in a cusp on the $x$-axis at infinity, implying zero drag for the latter case. The aftermost 124.21-degree case allowed is the only one of the infinite number of allowed separation points that has a cusped closure. The separation streamlines corresponding to any other point within the allowable range are open at infinity. The preceding cxample, which allowed the streakline method limited freedom in picking its own separation point from the five candidate points on the circular polygon, indicated a strong choice for the forwardmost 55.04-degree position. As a final test, the streakline method has been evaluated in application to the aftermost of the possible separation point positions allowed by the analytical solution for the smooth circular contour. The separation point is fixed at 125 degrees on the truncated circular polygon, shown in Fig. 3(b). At the steady flow limit, the shape of the contour inside the separation streamline is irrevelant because of the stagnant cavity flow. Truncation of the circle to the circular arc therefore represents no loss of generality in this regard. The primary

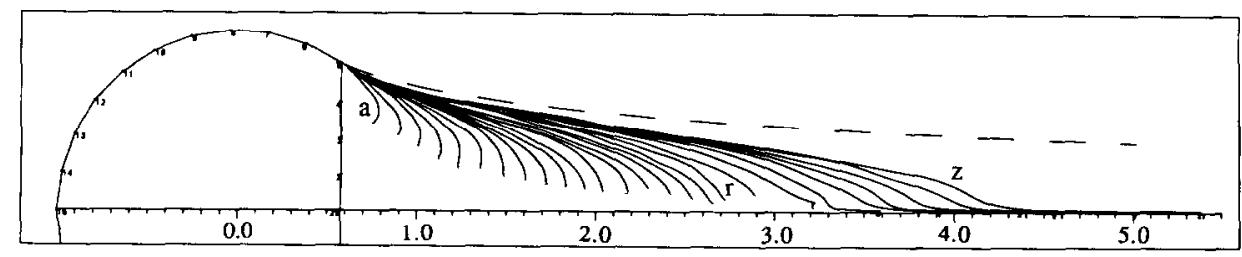

Fig. 10. Flow development past a circular arc. The letters a, r, and $z$ denote the time steps 220,3620 , and 5620 , respectively. The streaklines are shown at an interval of 200 time steps betwecn a and $\mathrm{r}$ and at an interval of 250 time steps between $r$ and $z$. 
interest in this example is to determine whether the streakline method will predict the cusped cavity predicted by the classical theory.

The computational parameters used with the model in Fig. $3(\mathrm{~b})$ are: $\Delta t=0.005, n_{\mathrm{avg}}=5$, $\Delta s_{\min }=0.02, \gamma_{\min }=0.05, r_{\delta}=0.02$, and $h=0.1$. The calculations are carried out for 5620 non-dimensional time steps. The streakline development is shown in Fig. 10. The time step numbers are indicated by the letters " $a$ ", " $r$ " and " $z$ " at the streakline ends. The corresponding step numbers are given in the caption.

The dashed line shown in Fig. 10 is the cusped analytical solution. It can be observed from Fig. 10 that at 3620 time steps the computed solution is continuously approaching the cusped analytical solution. However, after 3620 time steps it is not clear that the computed solution will form a cusp on the $x$-axis. The computations are carried further to 5620 time steps. The figure on an expanded scale (not shown) clearly shows the cusp formation on the $x$-axis from time step 4370 on. With the cusped cavity closing toward the $x$-axis, less time is required here in order to obtain a given degree of convergence toward the analytical solution. Immediately behind the body, the computed result is quite close to the analytical solution at 5620 time steps. The vortex strength of the streakline remains nearly constant over the entire length of the trailer except at the ends, where the strength drops off sharply. This is entirely consistent with the other cases presented in the preceding subsections.

\section{Conclusions}

The streakline method developed here is unique as to its formulation. The shed vortex sheets are true continuous sheets, represented by chains of rigid, hinge-connected vortex segments versus point vortices or unconnected vortex segments as used in other vortical flow formulations. The free vortex sheets, representing the separated body boundary layer, are convected in space by the velocity field acting through a kinematic boundary condition. New links are added at the downstream ends of the sheets as needed to contain the flowing vorticity. The vorticity flows along the sheets according to a dynamic boundary condition, which is actually the Helmholtz vorticity equation compressed into the infinitesimally thin rotational flow domain of the vortex sheets.

The streakline method views the flow as evolving on different scales in both space and time, but the method has the objective of retaining only the global features of the large scale flow existing at large values of time. The small scale flow details that tend to be so troublesome in attempts to forward step initial value Euler formulations to the steady state are avoided in the formulation by filtering the numerical data, in both space and time, as it evolves. This filtering is accomplished by computing the induced field velocities on a fixed grid covering the solution domain. The time filter is then applied by back-averaging the velocities at each field grid point over some number of successive time steps. The number of successive time steps reflects the filter band width. Spatial filtering of the flow details takes the form of a smoothing accomplished primarily by interpolating the field grid velocities back to the vortex shects in applying the boundary conditions versus the direct vorticity self-induction usually implemented. A cutoff radius between the free vortex sheets in the field and the field grid points is maintained to further smooth the field by limiting the velocity gradients induced by the evolving sheets.

The details of the formulation and computational procedure are covered in the first three sections. 
On specifying the separation point on the two-dimensional contour, it is demonstrated that the streakline method numerical solution evolves toward the Kirchhoff steady free-streamline flow as time progresses.

For polygonal body contours with "corners" where ideal flow separation can occur, the method allows the shedding of vortex sheets from any number of corners beginning at the initial time. Only one separation streamline, per side, can exist at the steady flow limit in two-dimensional symmetric free-streamline flows. The streakline method is essentially assigned the task of picking the correct separation point from the candidate points assigned on the polygonal contour. For the knife edge and the step, where both the separation points are allowed the freedom to shed, the streakline method picks the physically correct point, that being the forward point, in both cases.

The possibility of establishing the position of separation points on general two-dimensional polygonal contours from purely ideal flow considerations was investigated further by applying the streakline method to a semi-circular contour, modelled as a polygon. Here, shedding was allowed from five of the eleven polygon corner points. Vortex sheets separated initially from four of the five points. At the fifth point, on the forward face of the semi-circle, the vortex sheet chose to remain attached to the contour throughout the entire solution time. The vortex strengths of threc of the four frec field vortex shects that were shed initially ultimately decayed, or were decaying, to negligible levels when the computation was terminated. The single remaining vortex sheet is progressing, in both position and strength, toward the solution for the forwardmost separation point allowed by the classical free-streamline theory for the circle. The steady free-streamline solution for the circle is indeterminate as to the separation point position, establishing only the angular range of possible separation point positions over the circular contour. The forwardmost position, at 55.04 degrees from the forward stagnation point, corresponds to the maximum drag solution.

Returning to the flow past a circle, it is tempting to conceptually extrapolate the results found for the semi-circular polygon contour to general smooth contours by letting the flat polygon sides become infinitesimal in length but infinite in number. However, indications are that for smooth contours, the ability to achieve smooth separation from Euler equation formulations depends on the "artificial viscosity" created by numerical discretization. Such damping effects are created purposely in the streakline method by the temporal and spatial smoothing performed in the process of the computation.

The aftermost separation point position from the analytical solution for the circle, at 124.21 degrees, is the only position which gives a closed cavity downstream; all of the others correspond to open streamlines at infinity. This aftermost limiting case is a zero drag solution, as the free streamline closes in a cusp on the $x$-axis at infinity. This is the last analyzed case using the streakline method. Here the separation point was fixed at 125 degrees. It is clearly demonstrated that the cusped streamline, closing on the axis, is being captured by the streakline method numerical solution.

It is possible to extend this methodology to three dimensions, however, the modifications involved are not trivial. In two dimensions the free vortex sheets must realistically close in a slope singularity, reflecting the effects of turbulent dissipation in the wake. Open free-streamline solutions in two dimensions are therefore of limited practical value in themselves. In three dimensions, however, closure of the free vortex sheets is not required in view of the convection in the third direction. 


\section{Acknowledgement}

I would like to thank Professor William S. Vorus, my thesis advisor, for introducing me to this problem. This work was partly funded by the Office of Naval Research under the Accelerated Research Initiative grant number N14-86-k-0118. I would like to thank the Theoretical Flow Physics Branch at NASA Langley Research Center for supporting me under the contract number NAS1-18599 while writing this paper. I would also like to thank Dr. Chen-Huei Liu and another referee for their suggestions.

\section{References}

[1] G. Birkhoff and E.H. Zarantonello, Jets Wakes and Cavities (Academic Press, New York, 1957).

[2] S. Brodetsky, Discontinuous fluid motion past circular and elliptic cylinders, Royal Soc. Proc. 102 (1923) $542-553$.

[3] S.P.G. Dinavahi, A streakline method for computing vortical flows in two dimensions, Ph.D. Thesis, The University of Michigan, Ann Arbor, MI (1988).

[4] C.A. Ford, Discontinuous fluid motion past an elliptic barrier, Proc. Leeds Philos. Literary Soc. 1 (1928) 209-216.

[5] Sir Horace Lamb, Hydrodynamics (Dover, New York, 1945).

[6] T. Levi-Civita, Scie e leggi di resistenzia, Rend. Circ. Mat. Palermo 23 (1907).

[7] Lighthill Rosenhead, Laminar Boundary Layers (Oxford Universily Press, Oxford, 1963).

[8] R.W. Newsome, A comparison of Euler and Navier-Stokes solutions for supersonic flow over a conical delta wing, AIAA 23rd Aerospace Sciences Meeting, Reno, NV (1985).

[9] A. Rizzi and L. Eriksson, Computation of inviscid incompressible flow with rotation, J. Fluid Mech. 153 (1985).

[10] V.C. Schmieden, Hohlraumbildung in der idealen Flüssigkeit, Ann. Phys. (1927) 350-356.

[11] V.C. Schmicden, Dic unstetige Strömungen um einen Kreiszylinder, Ing. Arch. I (1929) 104109.

[12] V.C. Schmieden, Über die Eindeutigkeit der Lösungen in der Theorie der unstetigen Strömungen, Ing. Arch. III (1932) 356-370.

[13] V.C. Schmieden, Über die Eindeutigkeit der Lösungen in der Theorie der unstetigen Strömungen II, Ing. Arch. $\mathrm{V}$ (1934) 373-375. 